\title{
COVID-19-related mortality in cancer patients in an Irish setting
}

\author{
Anna Linehan ${ }^{1,2}$ - Orla Fitzpatrick ${ }^{1,2} \cdot$ Darren Cowzer $^{1,2} \cdot$ Maeve A. Hennessy ${ }^{1,2} \cdot$ Zac L. Coyne $^{1,2} \cdot$ Amy Nolan $^{1,2}$. \\ Maeve Clarke ${ }^{1,2} \cdot$ Roisin Ni Dhonaill ${ }^{1,2} \cdot$ Bryan T. Hennessy ${ }^{1,2} \cdot$ Patrick G. Morris ${ }^{1,2} \cdot$ Liam Grogan $^{1,2}$. \\ Oscar Breathnach ${ }^{1,2}$
}

Received: 5 February 2021 / Accepted: 18 September 2021 / Published online: 25 October 2021

(c) The Author(s), under exclusive licence to Royal Academy of Medicine in Ireland 2021

\begin{abstract}
Background The COVID-19 pandemic has impacted significantly on healthcare across the globe. It has been reported to have higher incidence and be associated with worse outcomes in patients with cancer.

Aim To examine the characteristics of patients with cancer who were diagnosed with COVID-19 and to identify factors which may predict a poorer outcome.

Methods Patients attending oncology services in Beaumont Hospital who were diagnosed with COVID-19 between March and May 2020 were included. Demographics and outcomes were determined by chart review.

Results Twenty-seven patients were included in the study. The median age was 62;59\% were male. Ten patients (37\%) died all of whom had metastatic or incurable locally advanced disease. Patients with lung cancer had a higher rate of COVID-19 and poorer outcomes. Those with a performance status (PS) $\geq 3$ were more likely to die than those with PS $\leq 2$. Compared to those who recovered, patients who died had a higher number of organs affected by cancer and a higher mean Palliative Prognostic Score.

Conclusion Patients attending oncology services during the initial phase of the COVID-19 pandemic had an increased rate of SARS-CoV-2 infection and a higher mortality rate than the general population. Those who died had more advanced cancer as demonstrated by poorer performance status, a greater burden of metastatic disease and a higher Palliative Prognostic Score.
\end{abstract}

Keywords Cancer $\cdot$ Chemotherapy $\cdot$ COVID-19 $\cdot$ Malignancy $\cdot$ Mortality $\cdot$ SARS CoV-2

\section{Introduction}

The COVID-19 pandemic has impacted significantly on mortality and healthcare systems across the globe. In Ireland, during the initial outbreak in the period from March to May 2020, there were almost 25,000 cases of SARS-CoV-2 infection reported and over 1600 deaths recorded. In early studies, from the initial centre of the epidemic in Wuhan, China, COVID-19 was reported to have a higher incidence in patients with cancer than in the general population [1-3]. It was also reported that patients with cancer had a higher risk of severe adverse events (including ventilation, intensive care admission and death) compared to those who did not

Anna Linehan

1 Department of Medical Oncology, Beaumont Hospital, Dublin, Ireland

2 Cancer Clinical Trials and Research Unit, Beaumont Hospital, Dublin, Ireland have cancer [1]. This leads to widespread changes in oncology practices worldwide.

Within the oncology community, there has been a drive to investigate factors which might impact outcomes of patients with cancer who contracted COVID-19. A large UK prospective study looking at 800 patients with a diagnosis of active cancer and symptomatic COVID-19 infection concluded that those who died were significantly older $(73$ years vs 66 years; $p<0.001$ ), more likely to be male than female (33\% vs. 20\%) and had higher numbers of comorbidities [3]. The COVID-19 and Cancer Consortium (CCC19) collected data from the USA, Canada and Spain reported similarly that age, male sex and more comorbidities were associated with an increased 30-day mortality and additionally identified smoking, Eastern Cooperative Oncology Group (ECOG) performance status of 2 or higher and presence of active cancer as risk factors [2].

Beaumont Hospital is a large Dublin-based teaching hospital which was at the centre of the initial COVID-19 
outbreak in Ireland. We aimed to examine the demographics and outcomes of patients undergoing cancer-directed treatments in Beaumont Hospital who were diagnosed with COVID-19 and to identify factors which predict a poorer outcome in these patients.

\section{Methods}

This was a retrospective study conducted over the time period 11th March to 15th May 2020, during the first wave of the COVID-19 pandemic. Guidance from the Beaumont Ethics Committee was sought. The opinion expressed was that presentation of service evaluations did not require specific committee approval, nor did the publication of the result. The audit was assessed within the Medical Oncology Service's internal ethical committee (the consultant body) who felt that the open disclosure of the effect of COVID-19 on our inpatients through publication was ethically correct.

Patients who were attending Beaumont Hospital for systemic anti-cancer therapy and diagnosed with COVID-19 infection were identified. This included patients in both inpatient and outpatient settings who were receiving were planned to receive or had received anti-cancer therapy in the previous 8 weeks. Patients were referred for testing by a member of the oncology team based on symptoms or by virtue of being a close contact to a known case. Patients who were attending the oncology day ward for treatment and received a positive test result in the community were also included. COVID-19 testing was performed using RTPCR (reverse transcriptase-polymerase chain reaction) from the nasopharyngeal sample to detect SARS-CoV-2. Patients with radiological and clinical features suggestive of a diagnosis of COVID-19 but without a positive RT-PCR test were excluded.

Patient demographics, cancer type, stage and ECOG performance status were obtained by chart review. Nature and timing of most recent anti-cancer therapy were obtained from electronic chemotherapy prescribing record. Laboratory values were obtained by electronic record review. Statistical analyses were performed using SPSS version 28.0.0.0. Difference in means was calculated using two-sample $t$-test. Association between categorical variables was assessed using chi-squared test. Results were deemed statistically significant if $p$-value was $<0.05$.

In order to estimate the cancer-related prognosis of patients, we used a validated tool known as the Palliative Prognostic Score (PAP). This is a cumulative score based on the following variables: clinician prediction of survival, Karnofsky Performance Score, presence or absence of anorexia, presence or absence of dyspnoea, and lymphocyte percentage of the total white cell count [4]. A score of $<5.5$ is associated with a 30-day survival of $>70 \%$, whereas a score of $\geq 11$ is associated with a 30 -day survival of $<30 \%$. This was calculated based on data from date of diagnosis of COVID-19. Deaths were recorded at point of data cut off on 15th May 2020.

\section{Results}

In total, 703 patients attended oncology services for cancerdirected treatment during the study period. Of these, 27 patients were diagnosed with COVID-19 infection based on RT-PCR. A further 4 patients who were diagnosed clinically with COVID-19 due to characteristic symptoms and radiology were not included in the study population. There was an overall incidence of $4 \%$ in our patient population. Nine patients had a positive result following COVID-19 swab on the day ward; 6 patients (22\%) did not require admission while 3 patients $(11 \%)$ required inpatient treatment. Five patients (19\%) were diagnosed with COVID-19 following presentation to the Emergency Department with symptoms. A further 13 patients (48\%) developed COVID-19 during an inpatient stay for another reason (Fig. 1).

The median age of the patient population was 62 years (range: $38-84$ years) with $22 \%$ of patients aged 70 or older. There was a higher proportion of male patients at $59 \%$, with $41 \%$ female. The most common cancer type was colorectal,

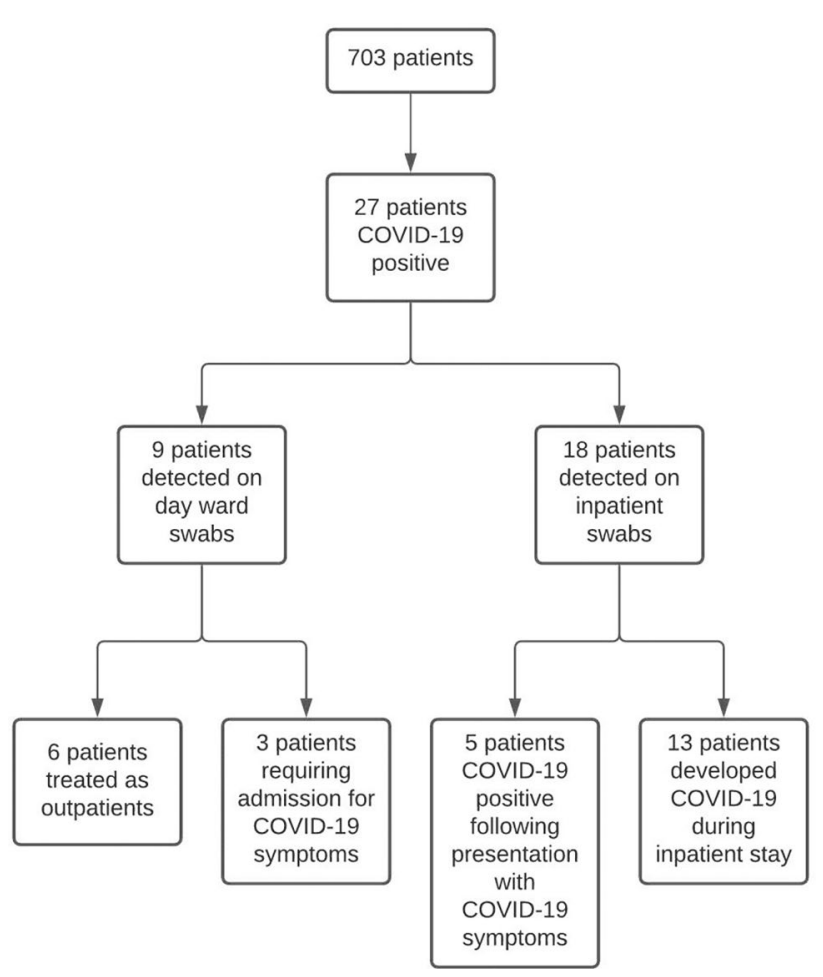

Fig. 1 Breakdown of patients attending oncology services who were diagnosed with COVID-19 based on RT-PCR 
representing almost one-third (30\%) of patients, followed by lung $(26 \%)$. The frequency of other cancer types is shown in Table 1. Seventeen patients (63\%) had metastatic disease, 4 $(15 \%)$ had locally advanced incurable disease, and 6 (22\%) were being treated with curative intent. None of the patients was admitted to critical care, and one patient received noninvasive ventilation. The most recent anti-cancer treatment for $19(70 \%)$ patients was chemotherapy; 11 (41\%) had received chemotherapy in the 30 days preceding diagnosis of COVID-19. Two (7\%) patients were on immunotherapy treatment, and both of these patients had treatment in the preceding 30 days.

In total, 37\% (10/27) of this patient population died during the study period. This included deaths which were attributed to COVID-19 and deaths from other causes including cancer. The mean age of those that died (mean $(M)=61.4$, standard deviation $(S D)=13$ ) was older than those that survived $(M=58.4, S D=12)$, but this was not statistically significant $(p=0.57)$. A similar proportion of male and female patients who contracted the virus subsequently died (37\%; $36 \%$ ). All of those who died had incurable locally advanced or metastatic disease. Those with locally advanced or metastatic disease were more likely to die than those being treated with curative intent (odds ratio (OR) 1.9 95\% CI 1.2-2.9). Lung cancer patients represented $60 \%$ of those that died, and having lung cancer was associated with an increased risk of dying compared with other cancers on univariate analysis $(O R=24,95 \%$ CI 2-260). The mean time from COVID-19 diagnosis to death was 9.5 days.

The majority (90\%) of patients in our cohort that died had an ECOG performance status of $\geq 3$ (Fig. 2). The mean ECOG performance status of those that died $(M=3.9$, $S D=2.3$ ) was significantly greater than that of those that survived $(M=1.9, S D=1.7, p<0.001)$. Those with an ECOG performance status $\leq 2$ had a reduced risk of death compared with those with PS $\geq 3$ (OR: 0.056; 95\% CI 0.008, 0.37). Patients that died also had more sites of metastatic disease. The mean number of organs involved by cancer was $3.9(S D=2.3)$ compared to $1.9(S D=1.7)$ in the group that survived. $(p=0.039)$ (Table 1). All patients who recovered had a Palliative Prognostic Score calculated at $<5.5$. In the group that died, 2 (20\%) had a PAP score of $<5.5,3(30 \%)$
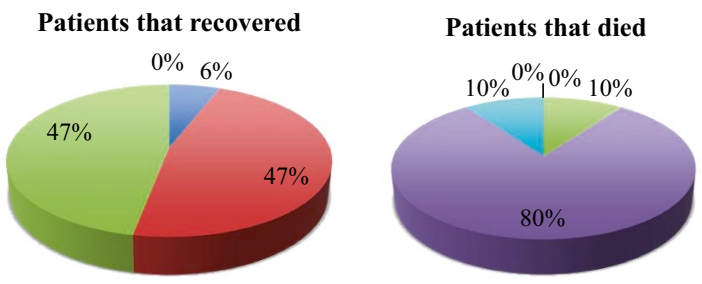

PS

Fig. 2 Performance status (PS) of patients that contracted COVID-19 and died compared with those who recovered had a PAP score of between 5.5 and 11, and $5(50 \%)$ had a score of $>11$ (Fig. 3). The mean PAP score for those that died was significantly higher than for those that survived $(M=10.4, S D=4.5$ versus. $M=1.5, S D=1.6, p<0.001)$.

With regard to recent anti-cancer treatment, 11 patients were treated with chemotherapy in the 30 days prior to COVID-19 diagnosis. Of these, 3 (27\%) died and 8 (73\%) survived. There were small numbers of patients on other treatments such as immunotherapy and hormonal therapy.

\section{Discussion}

In our study of patients with cancer who contracted COVID19 , we found an incidence of $4 \%$ which was higher than the incidence of $0.4 \%$ in the general population at this time point. The higher incidence in this population is not unexpected. These patients are at increased risk of exposure due to frequent hospital visits for treatment, especially considering most people were restricting their movements at this time. Many patients required inpatient hospital treatment increasing their risk of exposure. Treatment with cancerdirected therapies and drugs such as steroids can predispose to contracting infections. In addition, in order to protect patients attending oncology day services, patients were sent for testing if there was any clinical suspicion of COVID-19, and not all would have met the criteria for testing in the community at the time. International incidence figures in a cancer population range between 0.79 and $4.2 \%$ [1, 5-7].

The mortality rate of $37 \%$ is higher than that seen in other similar studies. This number includes all deaths that occurred in the patient population during the study period and therefore some of these were due to underlying

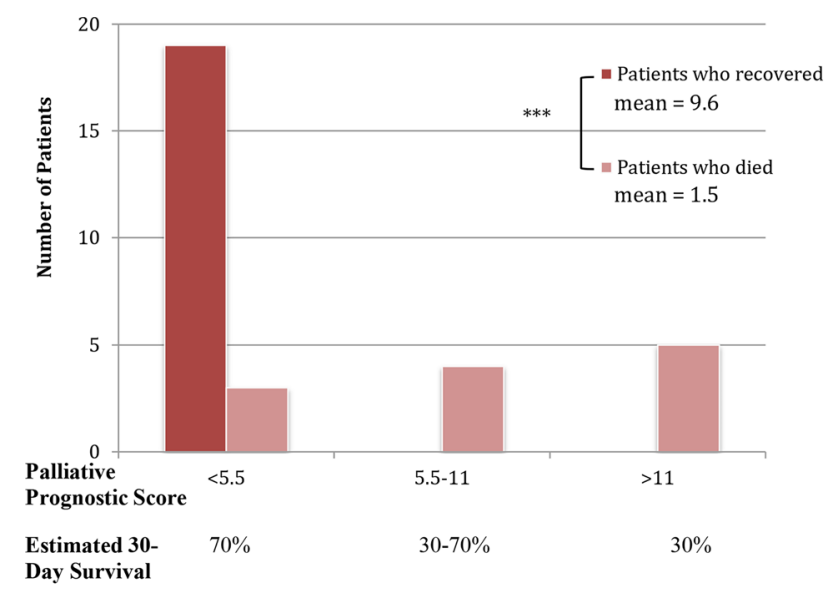

Fig. 3 Palliative Prognostic Scores (PAP) for patients with active cancer who contracted COVID-19. The mean PAP score for those that died was significantly higher than for those that survived (9.6 vs. 1.5, $p<0.001)$. $* * *$ denotes statistical significance 
Table 1 Characteristics of patients attending cancer services diagnosed with COVID-19

\begin{tabular}{|c|c|c|c|c|c|}
\hline & $\begin{array}{l}\text { All patients } \\
N=27(100 \%)\end{array}$ & $\begin{array}{l}\text { Patients who died } \\
N=10\end{array}$ & $\begin{array}{l}\text { Patients who } \\
\text { recovered } \\
N=17\end{array}$ & $\begin{array}{l}X^{2}(d f) \\
p \text {-value }\end{array}$ & $T$-test \\
\hline Age, median (range) & $62(38-81)$ & $65(38-81)$ & $61(38-77)$ & NA & $p=0.57$ \\
\hline Gender & & & & $0.004(1), 0.95$ & NA \\
\hline Male & $16(59 \%)$ & $6(60 \%)$ & $10(59 \%)$ & & \\
\hline Female & $11(41 \%)$ & $4(40 \%)$ & $7(41 \%)$ & & \\
\hline Cancer type & & & & $14.2(6), 0.27$ & NA \\
\hline Colorectal & $8(30 \%)$ & $1(10 \%)$ & $7(41 \%)$ & & \\
\hline Lung & $7(26 \%)$ & $6(60 \%)$ & $1(6 \%)$ & & \\
\hline Gastrooesophageal & $4(15 \%)$ & $1(10 \%)$ & $3(18 \%)$ & & \\
\hline CNS & $3(11 \%)$ & $0(0 \%)$ & $3(18 \%)$ & & \\
\hline Breast & $2(7 \%)$ & $0(0 \%)$ & $2(11 \%)$ & & \\
\hline Head and neck & $1(4 \%)$ & $1(10 \%)$ & $0(0 \%)$ & & \\
\hline Others & $2(7 \%)$ & $1(10 \%)$ & $1(6 \%)$ & & \\
\hline Cancer stage & & & & $5.62(2), 0.06$ & NA \\
\hline Metastatic & $17(63 \%)$ & $9(90 \%)$ & $8(47 \%)$ & & \\
\hline Locally advanced & $4(15 \%)$ & $1(10 \%)$ & $3(18 \%)$ & & \\
\hline Curative intent & $6(22 \%)$ & $0(0 \%)$ & $6(35 \%)$ & & \\
\hline $\begin{array}{l}\text { Sites of metastatic } \\
\text { disease, mean }(S D)\end{array}$ & $2.6(2.2)$ & $3.9(2.3)$ & $1.9(1.7)$ & NA & $p=0.039$ \\
\hline ECOG PS & & & & NA & $p<0.001$ \\
\hline $\mathbf{0}$ & $1(4 \%)$ & $0(0 \%)$ & $1(6 \%)$ & & \\
\hline 1 & $8(30 \%)$ & $0(0 \%)$ & $8(47 \%)$ & & \\
\hline 2 & $9(33 \%)$ & $1(10 \%)$ & $8(47 \%)$ & & \\
\hline 3 & $8(30 \%)$ & $8(80 \%)$ & $0(0 \%)$ & & \\
\hline 4 & $1(4 \%)$ & $1(10 \%)$ & $0(0 \%)$ & & \\
\hline Treatment & & & & $5.62(3), 0.13$ & NA \\
\hline Chemotherapy & $19(70 \%)$ & $5(50 \%)$ & $14(82 \%)$ & & \\
\hline Immunotherapy & $2(7 \%)$ & $1(10 \%)$ & $1(6 \%)$ & & \\
\hline Hormonal therapy & $1(4 \%)$ & $0(0 \%)$ & $1(6 \%)$ & & \\
\hline None & $5(19 \%)$ & $4(40 \%)$ & $1(6 \%)$ & & \\
\hline
\end{tabular}

Association between categorical variables was determined using chi-square analysis. Difference between means was determined using independent variable $t$-test

CNS central nervous system, DF degrees of freedom, ECOG PS Eastern Cooperative Group Performance Status, $S D$ standard deviation malignancy but may have been hastened by COVID-19. In an early Chinese study, the death rate in cancer patients was $28.6 \%$ [8]. This cohort included patients with a history of cancer, including up to 12 years prior. Similarly, in the UK Coronavirus Cancer Monitoring Project (UKCCMP), there was an all-cause mortality rate of $28 \%[3,8]$. However, that cohort was also broader, including patients treated with surgery, chemotherapy, or radiotherapy within the last 12 months. This may account for the difference in mortality rate. Additionally, there was a higher rate of intensive care unit admission in the UK study at $7 \%$, and they report that almost all deaths were attributed to COVID-19. In our population, some deaths were principally attributed to cancer rather than COVID-19.
In this study, patients who contracted COVID-19 and died did not have a statistically significant difference in age or sex than those who recovered. In other studies, male sex and older age were identified as risk factors for severe disease [2, $3,9]$. All of those who died had incurable locally advanced or metastatic cancer which has been shown to be risk factors for severe COVID-19 [2, 9, 10]. Those with lung cancer appeared more likely to contract COVID-19, with $11 \%$ (7 of a total of 63) patients with lung cancer attending oncology services receiving a positive test. Patients with gastrointestinal tract cancers also appeared to have a higher rate of COVID-19 positivity than other cancer types, with $8 \%$ of both patients with colorectal cancer (8/97) and gastrooesophageal cancer (4/51) who attended oncology services 
testing positive during the study period. The remaining cancers all had COVID-19 rates of $<5 \%$. Patients with lung cancer had poorer outcomes; however, the fact that lung cancer is the leading cause of cancer death must be taken into consideration and this accounts in part for the high odds ratio. Lung cancer patients have had poorer outcomes in other studies reported. $[2,3,9]$.

The principal finding from this study is that the population of patients with cancer who died following a positive COVID-19 test were largely those who had more advanced cancer. Those that died were more likely to have an ECOG performance status of $\geq 3$ than those that recovered and this was also seen in the CCC19 study [2]. Additionally, patients that died had a higher rate of organ involvement by cancer. The differences in Palliative Prognostic Score again reflect the severity of underlying illness with $50 \%$ of those who died having a score which was associated with a $70 \%$ mortality rate in the subsequent 30 days. These parameters all indicate that most of those patients who died were advanced stage of their malignant disease. This correlates with our knowledge of COVID-19 infection in cancer from other studies and also in patients without cancer, where increasing comorbidity has been shown to be associated with worse outcomes [11-13].

There did not appear to be associated between chemotherapy and increased risk of death in this patient population. The evidence from larger trials in this regard has thus far been conflicting. Two Chinese retrospective observational studies of 17 and 203 patients found that there was an increased risk of death with recent chemotherapy $[14,15]$. Subsequently, the UKCCMP study of 800 patients found that the administration of chemotherapy, immunotherapy or hormonal therapy within the last 4 weeks of life did not confer an increased risk of mortality [3]. Similarly, a review of 309 patients in a US hospital found that chemotherapy within 5 weeks of COVID-19 diagnosis was not associated with adverse COVID-19 outcomes [9].

There are several limitations to our study. The number of patients is small. This makes it difficult to draw definitive conclusions, results in large confidence intervals and makes sub-group analysis challenging. We likely did not identify all patients who contracted COVID-19. There were patients who had clinical and radiological features of COVID-19 and who were treated as such but did not have a positive COVID19 RT-PCR test, and it is possible that these were falsenegative results. Also, those who were mildly symptomatic or asymptomatic may not have been tested due to limited criteria for testing at the time. We included only patients who were on active treatment or planned to receive active treatment. As such, we may have identified a sicker cohort of patients than similar studies in the UK and China, and this makes it difficult to directly compare outcomes.

A significant limitation is that our analysis did not discriminate between causes of death. Many deaths were principally caused by cancer and the death certificates were not reviewed to determine what was deemed the leading cause of death. However, it is likely most of these deaths were multifactorial with both cancer and COVID-19 contributing. In addition, the calculation of the Palliative Prognostic Score required a clinician score and therefore is somewhat subjective.

\section{Conclusion}

Patients attending their regional oncology services during the initial phase of the COVID-19 pandemic had an increased rate of SARS-CoV-2 infection and a higher rate of death than the general population. Those who died all had incurable locally advanced or metastatic disease. They had more advanced cancer as demonstrated by a greater number of sites of metastatic disease, poorer performance status and a higher Palliative Prognostic Score. Patients with lung cancer appeared to have poorer outcomes. This Irish study reinforces that cancer patients are a high-risk group and should be protected in the setting of rising COVID-19 infections.

\section{Declarations}

Ethical approval All procedures performed in studies involving human participants were in accordance with the ethical standards of the institutional and/or national research committee and with the 1964 Helsinki declaration and its later amendments or comparable ethical standards.

Informed consent As this was a retrospective study, informed consent was not obtained.

Conflict of interest The authors declare no competing interests.

\section{References}

1. Liang W, Guan W, Chen R et al (2020) Cancer patients in SARSCoV-2 infection: a nationwide analysis in China. Lancet Oncol. https://doi.org/10.1016/S1470-2045(20)30096-6

2. Kuderer NM, Choueiri TK, Shah DP et al (2020) Clinical impact of COVID-19 on patients with cancer (CCC19): a cohort study. Lancet. https://doi.org/10.1016/S0140-6736(20)31187-9

3. Lee LYW, Cazier JB, Starkey T (2020) COVID-19 mortality in patients with cancer on chemotherapy or other anticancer treatments: a prospective cohort study. Lancet. https://doi.org/10.1016/ S0140-6736(20)31173-9

4. Maltoni M, Nanni O, Pirovano M et al (1999) Successful validation of the palliative prognostic score in terminally ill cancer patients. J Pain Symptom Manage. https://doi.org/10.1016/S08853924(98)00146-8

5. Bertuzzi AF, Marrari A, Gennaro N et al (2020) Low incidence of sars-cov-2 in patients with solid tumours on active treatment: an observational study at a tertiary cancer centre in lombardy. Italy Cancers (Basel). https://doi.org/10.3390/cancers12092352 
6. Rogado J, Obispo B, Pangua C et al (2020) Covid-19 transmission, outcome and associated risk factors in cancer patients at the first month of the pandemic in a Spanish hospital in Madrid. Clin Transl Oncol. https://doi.org/10.1007/s12094-020-02381-z

7. Yu J, Ouyang W, Chua MLK, Xie C (2020) SARS-CoV-2 Transmission in patients with cancer at a Tertiary Care Hospital in Wuhan. China JAMA Oncol. https://doi.org/10.1001/jamaoncol. 2020.0980

8. Zhang L, Zhu F, Xie L et al (2020) Clinical characteristics of COVID-19-infected cancer patients: a retrospective case study in three hospitals within Wuhan. China Ann Oncol. https://doi.org/ 10.1016/j.annonc.2020.03.296

9. Jee J, Foote MB, Lumish M et al (2020) Chemotherapy and COVID-19 outcomes in patients with cancer. J Clin Oncol. https:// doi.org/10.1200/jco.20.01307

10. Dai M-Y, Liu D, Liu M et al (2020) Patients with Cancer appear more vulnerable to SARS-CoV-2: a multi-center study during the COVID-19 outbreak. SSRN Electron J. https://doi.org/10.2139/ ssrn. 3558017

11. Wu Z, McGoogan JM (2020) Characteristics of and important lessons from the coronavirus disease (COVID-19) outbreak in China. JAMA. https://doi.org/10.1001/jama.2020.2648
12. Petrilli CM, Jones SA, Yang J et al (2020) Factors associated with hospital admission and critical illness among 5279 people with coronavirus disease, in New York City: Prospective cohort study. BMJ. https://doi.org/10.1136/bmj.m1966

13. Chow N, Fleming-Dutra K, Gierke R et al (2020) Preliminary estimates of the prevalence of selected underlying health conditions among patients with coronavirus disease 2019 - United States, February 12-March 28, 2020. MMWR Morb Mortal Wkly Rep 2020. https://doi.org/10.15585/mmwr.mm6913e2

14. Zhang H, Wang L, Chen $\mathrm{Y}$ et al (2020) Outcomes of novel coronavirus disease 2019 (COVID-19) infection in 107 patients with cancer from Wuhan. China Cancer 126:4023-4031. https://doi. org/10.1002/cncr.33042

15. Yang K, Sheng Y, Huang C et al (2020) Clinical characteristics, outcomes, and risk factors for mortality in patients with cancer and COVID-19 in Hubei, China: a multicentre, retrospective, cohort study. Lancet Oncol. https://doi.org/10.1016/S14702045(20)30310-7

Publisher's Note Springer Nature remains neutral with regard to jurisdictional claims in published maps and institutional affiliations. 